\title{
Brain Image Classification Based on Automated Morphometry and Penalised Linear Discriminant Analysis with Resampling
}

\author{
Eva Janousova, Daniel Schwarz \\ Masaryk University, Institute of \\ Biostatistics and Analyses, \\ Kamenice 3, 62500 Brno, \\ Czech Republic \\ Email: \{janousova, \\ schwarz\}@iba.muni.cz
}

\author{
Giovanni Montana \\ Imperial College London, \\ SW7 2AZ, London, \\ United Kingdom \\ Email: g.montana@imperial.ac.uk
}

\author{
Tomas Kasparek \\ Masaryk University, Department \\ of Psychiatry, Jihlavska 20, Brno, \\ Czech Republic \\ Email: tkasparek@fnbrno.cz
}

\begin{abstract}
This paper presents a new data-driven classification pipeline for discriminating two groups of individuals based on the medical images of their brain. The algorithm combines deformation-based morphometry and penalised linear discriminant analysis with resampling. The method is based on sparse representation of the original brain images using deformation logarithms reflecting the differences in the brain in comparison to the normal template anatomy. The sparse data enables efficient data reduction and classification via the penalised linear discriminant analysis with resampling. The classification accuracy obtained in an experiment with magnetic resonance brain images of first episode schizophrenia patients and healthy controls is comparable to the related state-of-the-art studies.
\end{abstract}

\section{INTRODUCTION}

$\mathrm{T}$ he last two decades have witnessed an explosive growth in the ability to "understand the human brain" a key to progress in neuroscience, to promote and protect brain health, and to develop treatments for restoring, regenerating, and repairing diseased brain functions. The motivations for that are clear: as populations inevitably grow older, mental disorders will increase dramatically - implying economic and social implications. The identification, characterization and validation of biomarkers for the major mental disorders would facilitate accurate prediction of disease risk, course, and therapeutic responses and ultimately lead to knowledge-based treatment and preventive strategies.

Computational neuroanatomy is a growing field of powerful applications of imaging modalities and computational techniques in neuroscience. It promises an automated methodology to characterize neuroanatomical configuration of structural magnetic resonance imaging (MRI) brain scans. One of the crucial techniques in this methodology is image registration. Its task is to find a spatial transformation which maps each point of an image onto its

This work was supported by the grant IGA MH CZ NR No. NT $13359-4 / 2012$ corresponding point of another image. Atlas-based registration is a special technique of computational neuroanatomy - not seen widely in other fields of biomedical imaging. It performs the task of spatial normalization of images according to a common reference anatomy termed as a brain atlas. Together with techniques adopted from inferential statistics and hypothesis testing, it allows to uncover brain regions with significant morphological differences between normal and clinical populations. Such techniques have been already used also in modern psychiatry research to seek for biomarkers and neurobiology of various mental diseases [1]-[4].

The real challenge for psychiatry is, however, to move from group analysis between patients and healthy volunteers to computer aided diagnostics on the level of an individual patient. Although pioneering works employing machine learning techniques have recently borne fruit in case of neurological diseases, this is extremely difficult in mental diseases. For instance, in schizophrenia - a disease with a complex neurobiology - the brain-imaging measurements in patients show considerable overlap with the normal range [5].

Algorithms, which have been proposed in the diagnostics of neurodegenerative disorders, relied on brain image data classification between patients and healthy controls. The most commonly used classification methods have been the linear discriminant analysis (LDA) [6]-[10], support vector machines (SVM) [11]-[13] or the k-nearest neighbour algorithm [14]-[16]. Due to the large amount of features obtained from 3-D medical images, the classification is often preceded by data reduction performed by principal component analysis [17], independent component analysis [18], selection of regions of interests (ROI) [6]-[8], and other data reduction methods. So far, only few studies have presented complex pipelines for data reduction and classification, such as the COMPARE method [19], which combines deformation-based morphometry with machine learning methods (watershed segmentation algorithm and support vector machine-recursive feature elimination 
technique). In [19] the COMPARE algorithm, classification of schizophrenia patients with very high classification accuracy $(91.8 \%$ for female subjects and $90.8 \%$ for male subjects) was applied. Thus, the complex pipeline seems to enable classification with a higher efficiency than other commonly used methods that have reported classification accuracy between $70 \%$ and $90 \%$ [5]-[18].

The aim of this paper is to present a new data-driven complex classification pipeline consisting of deformationbased morphometry (DBM) and penalised linear discriminant analysis (pLDA) with resampling. The DBMpLDA algorithm enables efficient data reduction and subsequent classification as it is based on sparse representation of the original image data. The algorithm starts with an application of the DBM on original MRI data to create 3-D deformations, which are then log-transformed and used in the pLDA with resampling to identify the brain regions with different local volumes in patients and controls. The last step comprises of classifying the brain images into groups of patients and healthy controls based on the features representing automatically detected brain regions.

The pLDA has been successfully employed in our previous imaging-genetics study [20] in which image phenotypes, using pre-selected pLDA, were used for searching the genotypes most associated with Alzheimer's disease. To our knowledge, this is the first study that uses pLDA for selecting brain imaging features for the purposes of distinguishing diseased individuals from healthy controls in schizophrenia research.

The rest of the paper is organized as follows. In Section II, we describe all the necessary steps in the classification pipeline. Section III shows the application of the proposed DBM-pLDA algorithm on T1-weighted MRI data of firstepisode schizophrenia patients and healthy controls. Section IV discusses the results and concludes the paper.

\section{METHODS}

The proposed data-driven algorithm for image classification is based on combining the deformation-based morphometry and penalised linear discriminant analysis with resampling. In the DBM, high-resolution nonlinear registration [21] of 3-D brain images with a digital brain atlas is performed. The resulting 3-D deformations represented by the displacement fields or their Jacobian determinants clearly show how the brain anatomy of a subject differs from the normal template anatomy in terms of local volume contractions and expansions. After logarithmic transformation, the 3-D deformations tend to be sparse, i.e. numerous voxels have zero or close to zero values. The sparse representation of the image data together with pLDA with resampling leads to effective selection of the most discriminating regions, as explained below. The selected brain voxels are then used as features in image classification. The DBM and pLDA are described in more details in the following two subchapters.

\section{A. Deformation-Based Morphometry}

Here, a brief summary is given on our algorithm for highresolution deformation-based morphometry with the underlying registration method, based on a spatial deformation model that allows for large deformations while preserving the topology of the images. Details can be found in [21].

The registration method operates directly on image intensity values with no data reduction by segmentation or classification. The 3-D displacement field which maximizes global mutual information between a reference image and a floating image is searched in an iterative process that involves computation of the local forces as a gradient of point similarity measures and their regularization using the spatial deformation model. The regularization involves two Gaussian spatial filters forming the combined elasticincremental model [22]. The first spatial filter regularizes displacement improvements that are proportional to the applied forces. These displacements are integrated into the final deformation, which is done iteratively by summation. The second part of the model represents the property of elastic materials in which displacements wane upon retracting the forces. This is ensured by a second Gaussian smoother. The resulting deformations preserve the topology of the images, i.e. only one-to-one mappings, termed diffeomorphic, are obtained. This requirement is satisfied by controlling the standard deviations of the Gaussian filters that affect the behaviour of the spatial deformation model. Standard deviations are incremented each time the minimum Jacobian determinant drops below a predefined threshold. The deformation should capture subtle anatomical variations among the studied images; therefore, the standard deviations of the Gaussians are decremented as well whenever the minimum Jacobian determinant starts rising during the registration process.

\section{B. Penalised Linear Discriminant Analysis with Resampling}

Prior to the penalised linear discriminant analysis with resampling, the logarithms of the Jacobian determinants computed from 3-D deformation fields are transformed into 1-D vectors and arranged in $(n \times p)$ matrix $\mathbf{X}$, where $n$ is the number of individuals in a data set and $p$ is the number of voxels in each deformation. All columns of the matrix $\mathbf{X}$ are mean-centred and have unit variance. It is assumed that all $n$ individuals have been labelled as one of the two classes, which are denoted by $D$ (diseased individuals) and $H$ (healthy controls). The number of individuals in each class is $n_{D}$ and $n_{H}$, respectively, and $n=n_{D}+n_{H}$.

The common LDA aims at finding a direction vector $\mathbf{v}$ that best discriminates two classes within a data sample via maximizing the between-class variance and simultaneous minimizing of the within-class variance. The between-class scatter matrix, denoted as $\mathbf{S}_{B}$, is calculated as: 


$$
\mathbf{S}_{B}=\left(\mathbf{m}_{H}-\mathbf{m}_{D}\right)^{T}\left(\mathbf{m}_{H}-\mathbf{m}_{D}\right),
$$

where $\mathbf{m}_{H}=\frac{1}{n_{H}} \sum_{i=1}^{n_{H}} \mathbf{x}_{i .}$ is the mean vector of class $H$, $\mathbf{m}_{D}=\frac{1}{n_{D}} \sum_{i=1}^{n_{D}} \mathbf{x}_{i .}$ is the mean vector of class $D$ and $\mathbf{x}_{i .}, i=1, \ldots, n$, are rows of the matrix $\mathbf{X}$.

The within-class scatter matrix, denoted as $\mathbf{S}_{W}$, is defined as:

$$
\begin{aligned}
\mathbf{S}_{W} & =\sum_{i=1}^{n_{H}}\left(\mathbf{x}_{i .}-\mathbf{m}_{H}\right)^{T}\left(\mathbf{x}_{i .}-\mathbf{m}_{H}\right) \\
& +\sum_{i=1}^{n_{D}}\left(\mathbf{x}_{i .}-\mathbf{m}_{D}\right)^{T}\left(\mathbf{x}_{i .}-\mathbf{m}_{D}\right) .
\end{aligned}
$$

The direction vector $\mathbf{v}$ is then a solution of the following optimization problem:

$$
\max _{\mathbf{v}}\left\{\mathbf{v}^{T} \mathbf{S}_{B} \mathbf{v}\right\} \text { subject to } \mathbf{v}^{T} \mathbf{S}_{W} \mathbf{v}=1 \text {. }
$$

In the penalised LDA [23], a penalty is imposed on the $l_{1}$ norm of the direction vector $\mathbf{v}$ which leads to setting of coefficients $\mathbf{v}_{j}, j=1, \ldots, p$, of the least discriminative features to zero. If the input data into the pLDA are already sparse, the amount of selected features (i.e. features with non-zero coefficients) is smaller and the classification results tend to be more stable than while using original brain images in the data analysis. In pLDA, the optimisation problem changes to:

$$
\max _{\mathbf{v}}\left\{\mathbf{v}^{T} \mathbf{S}_{B} \mathbf{v}-\lambda \sum_{j=1}^{p} s_{j}\left|v_{j}\right|\right\} \text { subject to } \mathbf{v}^{T} \mathbf{S}_{W}^{*} \mathbf{v}=1,(4)
$$

where $\mathbf{S}_{W}^{*}$ is the diagonal estimate of $\mathbf{S}_{W}$, $\operatorname{diag}\left(\mathbf{S}_{W}\right)=\left(s_{1}^{2}, \ldots, s_{p}^{2}\right)$ and $\lambda$ is the regularization parameter that controls the number of selected features. Specifically, when $\lambda$ is exactly zero, no penalty is imposed and all $p$ features contribute in the direction vector $\mathbf{v}$. As $\lambda$ increases from zero, less features contribute in $\mathbf{v}$. At its maximum value $\lambda_{\text {max }}$, all coefficients of $\mathbf{v}$ are set to zero. A common approach for tuning $\lambda$ involves cross-validating the prediction error for a grid of values of $\lambda$ and selecting the value of $\lambda$ that leads to the smallest cross-validated error. However, this approach may be prone to sampling errors. Therefore, we opted for a resampling method proposed in [24] for sparse predictive modelling. This procedure aims to calculate selection probabilities for each feature by repeatedly fitting the pLDA model on random subsets of the data set, while keeping track of the features associated to non-zero coefficients of $\mathbf{v}$. The final set of the most discriminative features consists of voxels with selection probability higher than 0.5 .
It should be noted that by using the data resampling, a set of features over the range $\left[\lambda_{\min }, \lambda_{\max }\right]$ with stable classification results is selected instead of tuning the regularization parameter $\lambda$ [24]. The final set of selected features is then used for classification of individuals into the class $D$ or $H$ using LDA.

\section{EXPERIMENT AND RESULTS}

\section{A. Imaging Data Used in the Experiment}

The proposed classification pipeline was tested in an experiment with magnetic resonance brain imaging data of 52 male patients with first-episode schizophrenia and 52 sex- and age-matched healthy control subjects. The median age of the patients and controls was 22.9 years (range 17-40 years) and 23.0 (range 18-38 years), respectively. Thirtynine patients and the same number of controls took part in our previous study [25]. All subjects signed an informed consent before entering the study.

All 52 patients were recruited from males hospitalised in the all-males unit of the Department of Psychiatry, Masaryk University in Brno for first-episode schizophrenia. The diagnosis was established during a clinical interview guided by the International Statistical Classification of Disease and Related Health Problems (ICD-10) research criteria and was focused on information about the family and personal history, the somatic conditions, substance abuse, pharmacological history and the current treatment, previous psychiatric conditions and, finally, on the current clinical manifestation, the presenting symptoms, the duration, and the functional impact. Next, the patients were physically examined, including blood (haematology and biochemistry) and urine analysis (biochemistry and toxicology). If abnormal findings were present, their origin was traced by additional examination. A fully trained senior psychiatrist (board certified in psychiatry) reviewed all information, established the diagnosis and suggested the case for inclusion in the study. MRI examination was performed during the first episode, that is, all patients were treated with antipsychotics for 3-14 weeks only at the time of MRI.

The 52 healthy control subjects without substance dependence, family or personal history of axis I psychiatric conditions, neurological or somatic conditions affecting the structure or function of the brain, and contraindications for MRI examination, were recruited from the community, the local staff and medical students.

All subjects were scanned with 1.5T Siemens Symphony machine. Whole head T1-weighted images were obtained using 3-D acquisition with IR/GR sequence, TR $1700 \mathrm{~ms}$, TE $3.93 \mathrm{~ms}$, TI $1100 \mathrm{~ms}$, flip angle $15^{\circ}$, 160 slices, voxel size $1.17 \times 0.48 \times 0.48 \mathrm{~mm}$, FOV $246 \times 246 \mathrm{~mm}$, and matrix size $512 \times 512$ voxels.

The 3-D T1-weighted images were checked for abnormalities and then pre-processed using SPM8 (http://www.fil.ion.ucl.ac.uk/spm/). Specifically, the images 


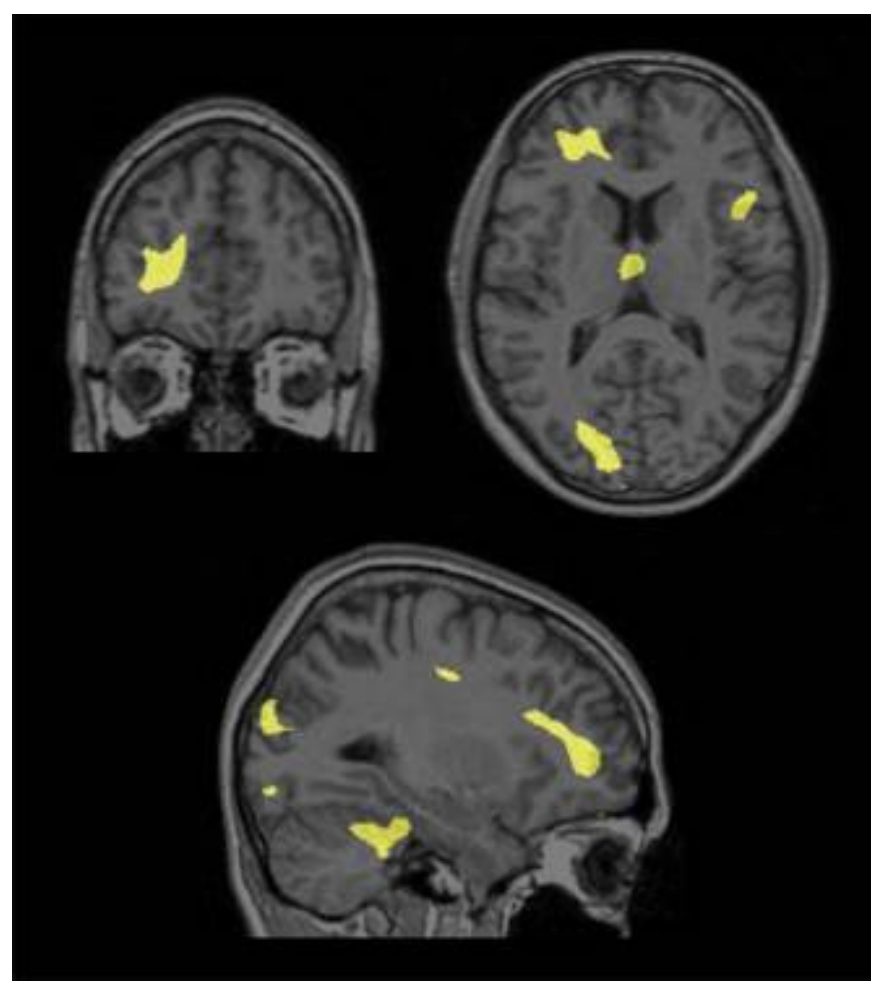

Fig. 1 Coronal, transversal and sagittal slices showing the automatically detected highly discriminative voxels (in yellow).

were corrected for bias-filed inhomogeneity and spatially normalised, i.e. transformed into stereotactic space.

\section{B. Classification efficiency}

The DBM-pLDA algorithm was applied on the preprocessed images. The classification efficiency of the DBMpLDA algorithm was evaluated with the leave-one-out cross-validation technique to avoid biased results. Consecutively, each of the $n$ subjects was selected as a testing subject and the remaining $n-1$ subjects were used for training the classifier. The testing subject was classified into the patient or healthy control class. Then, the resulting class was compared to the true classification label. The classification performances for all subjects were combined in order to create the overall classification performance measures, namely accuracy, sensitivity, specificity and precision, defined as:

$$
\begin{gathered}
\text { sensitivity }=\frac{T P}{T P+F N}, \\
\text { specificity }=\frac{T N}{T N+F P}, \\
\text { accuracy }=\frac{T P+T N}{T P+T N+F P+F N}, \\
\text { precision }=\frac{T P}{T P+F P},
\end{gathered}
$$

TABLE I

NUMBER OF SELECTED VOXELS AND CROSS-VALIDATED CLASSIFICATION PERFORMANCE MEASURES IN PERCENTAGE FOR VARIOUS $\lambda_{\text {in }}$ VALUES

\begin{tabular}{lcccc}
\hline \hline & $\lambda_{\min }=0.3$ & $\lambda_{\min }=0.4$ & $\lambda_{\min }=0.5$ & $\lambda_{\min }=0.6$ \\
\hline \# voxels & 315,123 & 107,967 & 30,461 & 5,113 \\
\hline Accuracy & 82.7 & 84.6 & 85.6 & 83.7 \\
\hline Sensitivity & 84.6 & 86.5 & 84.6 & 78.8 \\
\hline Specificity & 80.8 & 82.7 & 86.5 & 88.5 \\
\hline Precision & 81.5 & 83.3 & 86.3 & 87.2 \\
\hline \hline
\end{tabular}

where $T P, T N$ represent numbers of true positive and true negative results respectively, and $F P, F N$ represent numbers of false positive and false negative results respectively.

\section{Experiment Results}

The performance measures obtained in the classification of 104 schizophrenia patients and healthy controls are summarized in Table I. Our results demonstrate that the classification accuracy is stable for meaningful ranges of $\lambda$, i.e. ranges leading to an adequate number of selected voxels. In this experiment, $\lambda_{\max }$ was fixed to 0.9 and so, the table shows the results for the ranges $\left[\lambda_{\min }, 0.9\right]$. The best crossvalidated classification accuracy was $85.6 \%$ (sensitivity $84.6 \%$, specificity $86.5 \%$ ) while selecting about 30,000 most discriminative voxels. The selected features composed of the most discriminating voxels are shown in Fig. 1. They form connected regions in the left prefrontal cortex, the right anterior insula, the medial parts of the thalamus, and the cerebellar cortex.

In order to compare the proposed DBM-pLDA algorithm to other classification methods, the leave-one-out crossvalidation procedure was carried also with other classifiers, which have been used on neuroimaging data frequently: (i) LDA and (ii) SVM with linear kernel. Features for these classifiers were selected with the use of mass univariate analysis (Student's t-test, $p<0.01$ ), so that only the significant local volume changes in patients when compared to healthy controls were input to the classifiers. The resulting classification performance measures are summarized in Table II. The results show an improved classification efficiency of the proposed DBM-pLDA algorithm, when compared to both DBM-LDA and DBMSVM algorithms.

\section{DISCUSSION AND CONCLUSIONS}

A classification pipeline for discriminating two groups of individuals based on brain images has been presented. The fully automated data-driven algorithm consists of deformation-based morphometry and penalised linear discriminant analysis with resampling. Firstly, sparse representation of the original brain images using logarithms of deformations, that are the results of high-dimensional nonlinear registration of the brain images with a digital brain atlas, is acquired. Secondly, the sparse data is reduced using 
TABLE II

CLASSIFICATION EFFICIENCY OF THE PROPOSED DBM-PLDA ALGORITHM COMPARED TO DBM-LDA AND DBM-SVM ALGORITHMS.

\begin{tabular}{lcccc}
\hline \hline & Accuracy & Sensitivity & Specificity & Precision \\
\hline DBM-pLDA & 85.6 & 84.6 & 86.5 & 86.3 \\
\hline DBM-LDA & 66.3 & 59.6 & 73.1 & 68.9 \\
\hline DBM-SVM & 70.2 & 69.2 & 75.0 & 73.5 \\
\hline \hline
\end{tabular}

pLDA with resampling and then classified into the group of patients or healthy controls.

To our knowledge, this is for the first time when pLDA is used for selecting highly discriminative brain imaging features in schizophrenia. In our previous study, pLDA was successfully applied for selecting image phenotypes that were used in searching for genotypes most associated with Alzheimer's disease [20]. Here, pLDA is used in combination with DBM in the pipeline for the classification of MRI data of patients with first episode schizophrenia (FES) and healthy controls. Only FES patients were used in this study as it is known that longer duration of the illness leads to higher magnitude of morphological changes in the brain of schizophrenia patients [26]. Thus, the classification results can be overestimated if the data set contains chronic schizophrenia patients as well. To avoid biased results, only males were used in the analysis, as it was shown that there are differences in structural brain abnormalities in males and females [27].

The proposed algorithm uses the whole volume of the brain and is fully automated. Thus, it overcomes limitations of traditional ROI-based classification analyses. The ROIbased methods need prior knowledge about the regions that might be affected by the disease. As schizophrenia is a large-scale disorder of neurocognitive networks rather than confined to specific regions [1]-[4], whole brain analysis is more appropriate. Moreover, the fully automated method is less time consuming and error-prone than manual tracing of the ROIs.

High classification accuracy $(85.6 \%)$ has been achieved while applying the proposed DBM-pLDA algorithm on T1weighted MRI data of first-episode schizophrenia patients and healthy controls. The efficiency of the algorithm is comparable or superior to the other state-of-the-art studies dealing with the classification of schizophrenia patients [5][18]. However, the classification performance is smaller than in [19], in which the COMPARE algorithm enabled classification of schizophrenia and healthy females with accuracy equal to $91.8 \%$ and the classification of diseased and healthy males with accuracy of $90.8 \%$. Nevertheless, Fan et al. [19] used a mixed data set of patients with firstepisode schizophrenia and chronic schizophrenia. The fact that the morphological changes in chronic schizophrenia patients progress during the course of the disease, while the first episode schizophrenia is characterized only by subtle morphological abnormalities, could overestimate the results reported in [19].
A by-product of the classification pipeline is a selection of the most discriminating brain morphological features between patients and controls. The automatically detected discriminating brain regions were located in the left prefrontal cortex, the right anterior insula, the medial parts of the thalamus, and the cerebellar cortex. These results are consistent with those published in previous studies [1]-[4]. Moreover, it is known that these brain areas are involved in higher cognitive, integrative and regulatory functions that are impaired in schizophrenia [1]-[4].

Even though the results are promising, further experiments are necessary to investigate whether the DBMpLDA algorithm can assist in the early diagnosis of schizophrenia. A limitation of this study might be the slightly limited sample size, as Nieuwenhuis et al. [12] recommend to use more than 130 subjects. However, our data set containing 104 subjects is larger than the data sets used in most of the schizophrenia studies [5]-[8], [11], [14][15], [17]-[19]. The next step in our research will be a replication using a completely independent set of schizophrenia subjects. We would also like to test the performance of the algorithm on other patient groups.

In conclusion, the associations between the automatically detected discriminating morphology features and their significance in the neurobiology of schizophrenia, as well as the high accuracy of classification of patients and healthy controls, demonstrate the face validity of our approach that combines DBM and pLDA with resampling.

\section{REFERENCES}

[1] I. C. Wright, S. Rabe-Hesketh, P. W. R. Woodruff, A. S. David, R. M. Murray, and E. T. Bullmore, "Meta-analysis of regional brain volumes in schizophrenia," Am. J. Psychiatry, vol. 157, no. 1, pp. 16-25, Jan. 2000 .

[2] M. E. Shenton, C. C. Dickey, M. Frumin, and R. W. McCarley, "A review of MRI findings in schizophrenia," Schizophr. Res., vol. 49, no. 1-2, pp. 1-52, Apr. 2001.

[3] M. A. Niznikiewicz, M. Kubicki, and M. E. Shenton, "Recent structural and functional imaging findings in schizophrenia," Curr. Opin. Psychiatry, vol. 16, no. 2, pp. 123-147, Mar. 2003.

[4] R. Honea, T. J. Crow, D. Passingham, and C. E. Mackay, "Regional deficits in brain volume in schizophrenia: A meta-analysis of voxelbased morphometry studies," Am. J. Psychiatry, vol. 162, no. 12, pp. 2233-2245, Dec. 2005.

[5] D. Sun, T. G. M. van Erp, P. M. Thompson, C. E. Bearden, M. Daley, L. Kushan, M. E. Hardt, K. H. Nuechterlein, A. W. Toga, and T. D. Cannon, "Elucidating a Magnetic Resonance Imaging-Based Neuroanatomic Biomarker for Psychosis: Classification Analysis Using Probabilistic Brain Atlas and Machine Learning Algorithms," Biol. Psychiatry, vol. 66, no. 11, pp. 1055-1060, Dec. 2009.

[6] C. M. Leonard, J. M. Kuldau, J. I. Breier, P. A. Zuffante, E. R. Gautier, D. C. Heron, E. M. Lavery, J. Packing, S. A. Williams, and C. A. DeBose, "Cumulative effect of anatomical risk factors for schizophrenia: An MRI study," Biol. Psychiatry, vol. 46, no. 3, pp. 374-382, Aug. 1999.

[7] K. Nakamura, Y. Kawasaki, M. Suzuki, H. Hagino, K. Kurokawa, T. Takahashi, L. Niu, M. Matsui, H. Seto, and M. Kurachi, "Multiple structural brain measures obtained by three-dimensional magnetic resonance imaging to distinguish between schizophrenia patients and normal subjects," Schizophr. Bull., vol. 30, no. 2, pp. 393-404, 2004.

[8] Y. Takayanagi, Y. Kawasaki, K. Nakamura, T. Takahashi, L. Orikabe, E. Toyoda, Y. Mozue, Y. Sato, M. Itokawa, H. Yamasue, K. Kasai, M. Kurachi, Y. Okazaki, M. Matsushita, and M. Suzuki, "Differentiation 
of first-episode schizophrenia patients from healthy controls using ROI-based multiple structural brain variables," Prog. Neuropsychopharmacol. Biol. Psychiatry, vol. 34, no. 1, pp. 10-17, Feb. 2010

[9] M. Ota, N. Sato, M. Ishikawa, H. Hori, D. Sasayama, K. Hattori, T. Teraishi, S. Obu, Y. Nakata, K. Nemoto, Y. Moriguchi, R. Hashimoto, and H. Kunugi, "Discrimination of female schizophrenia patients from healthy women using multiple structural brain measures obtained with voxel-based morphometry," Psychiatry Clin. Neurosci., vol. 66, no. 7, pp. 611-617, Dec. 2012.

[10] E. Janousova, D. Schwarz, and T. Kasparek, "Combining various types of classifiers and features extracted from magnetic resonance imaging data in schizophrenia recognition," Psychiatry Res. Neuroimaging, to be published.

[11] K. M. Pohl and M. R. Sabuncu, "A Unified Framework for MR Based Disease Classification," in Information Processing in Medical Imaging, Proceedings, vol. 5636, J. L. Prince, D. L. Pham, and K. J. Myers, Eds. Berlin: Springer-Verlag Berlin, 2009, pp. 300-313.

[12] M. Nieuwenhuis, N. E. M. van Haren, H. E. H. Pol, W. Cahn, R. S. Kahn, and H. G. Schnack, "Classification of schizophrenia patients and healthy controls from structural MRI scans in two large independent samples," Neuroimage, vol. 61, no. 3, pp. 606-612, Jul. 2012.

[13] P. Dluhos, D. Schwarz, and T. Kasparek, "Wavelet features for recognition of first episode of schizophrenia from MRI brain images," Radioengineering, vol. 23, pp. 274-281, Apr. 2014.

[14] Y. X. Liu, L. Teverovskiy, O. Carmichael, R. Kikinis, M. Shenton, C. S. Carter, V. A. Stenger, S. Davis, H. Aizenstein, J. T. Becker, O. L. Lopez, and C. C. Meltzer, "Discriminative MR image feature analysis for automatic schizophrenia and Alzheimer's disease classification," in Medical Image Computing and Computer-Assisted Intervention Miccai 2004, vol. 3216, C. Barillot, D. R. Haynor, and P. Hellier, Eds. Berlin: Springer-Verlag Berlin, 2004, pp. 393-401.

[15] P. Wang and R. Verma, "On Classifying Disease-Induced Patterns in the Brain Using Diffusion Tensor Images," in Medical Image Computing and Computer-Assisted Intervention - Miccai 2008, vol. 5241, D. Metaxas, L. Axel, G. Fichtinger, and G. Szekely, Eds. Berlin: Springer-Verlag Berlin, 2008, pp. 908-916.

[16] D. Schwarz and T. Kasparek, "Brain morphometry of MR images for automated classification of first-episode schizophrenia,“ Information Fusion, vol. 19, pp. 97-102, Sep. 2014.
[17] F. Shi, Y. Liu, T. Jiang, Y. Zhou, W. Zhu, J. Jiang, H. Liu, and Z. Liu, "Regional homogeneity and anatomical parcellation for fMRI image classification: Application to schizophrenia and normal controls," in Medical Image Computing and Computer-Assisted InterventionMICCAI 2007, vol. 4792, N. Ayache, S. Ourdelin, and A. Maeder, Eds. Berlin: Springer-Verlag Berlin, 2007, pp. 136-143.

[18] O. Demirci, V. P. Clark, and V. D. Calhoun, "A projection pursuit algorithm to classify individuals using fMRI data: Application to schizophrenia," Neuroimage, vol. 39, no. 4, pp. 1774-1782, Feb. 2008.

[19] Y. Fan, D. Shen, R. C. Gur, R. E. Gur, and C. Davatzikos, "COMPARE: Classification of morphological patterns using adaptive regional elements," IEEE Trans. Med. Imaging, vol. 26, no. 1, pp. 93105, Jan. 2007.

[20] M. Vounou, E. Janousova, R. Wolz, J. L. Stein, P. M. Thompson, D. Rueckert, and G. Montana, "Sparse reduced-rank regression detects genetic associations with voxel-wise longitudinal phenotypes in Alzheimer's disease," Neuroimage, vol. 60, no. 1, pp. 700-716, Mar. 2012.

[21] D. Schwarz, T. Kasparek, I. Provaznik, and J. Jarkovsky, "A deformable registration method for automated morphometry of MRI brain images in neuropsychiatric research," IEEE Trans. Med. Imaging, vol. 26, no. 4, pp. 452-461, Apr. 2007.

[22] P. Rogelj and S. Kovacic, "Spatial deformation models for non-rigid image registration," in Proceedings of the 9th Computer Vision Winter Workshop (CVWW'04), Piran (Slovenia), 2004, pp. 79-88.

[23] D. M. Witten and R. Tibshirani, "Penalized classification using Fisher's linear discriminant," J. R. Stat. Soc. Ser. B - Stat. Methodol., vol. 73, pp. 753-772, 2011.

[24] N. Meinshausen and P. Bühlmann, "Stability selection," J. R. Stat. Soc. Ser. B - Stat. Methodol., vol. 72, pp. 417-473, 2010.

[25] T. Kasparek, C. E. Thomaz, J. R. Sato, D. Schwarz, E. Janousova, R. Marecek, R. Prikryl, J. Vanicek, A. Fujita, and E. Ceskova, "Maximum-uncertainty linear discrimination analysis of first-episode schizophrenia subjects," Psychiatry Res.-Neuroimaging, vol. 191, no. 3, pp. 174-181, Mar. 2011.

[26] I. Ellison-Wright, D. C. Glahn, A. R. Laird, S. M. Thelen, and E. Bullmore, "The anatomy of first-episode and chronic schizophrenia: An anatomical likelihood estimation meta-analysis," Am. J. Psychiatry, vol. 165, no. 8, pp. 1015-1023, Aug. 2008.

[27] H. Nasrallah, S. Schwarzkopf, S. Olson, and J. Coffman, "Gender Differences in Schizophrenia on Mri Brain-Scans," Schizophr. Bull., vol. 16, no. 2, pp. 205-209, 1990. 